\title{
Nucleotide Metabolic Process
}

National Cancer Institute

\section{Source}

National Cancer Institute. Nucleotide Metabolic Process. NCI Thesaurus. Code C19403.

Biosynthesis and catabolism of nucleic acids and their subunits 